\title{
Efficacy and patient satisfaction regarding lymphovenous bypass with sleeve-in anastomosis for extremity lymphedema
}

\author{
Jae-Ho Chung, Si-Ook Baek, Ho-Jin Park, Byung-Il Lee, Seung-Ha Park, Eul-Sik Yoon \\ Department of Plastic Surgery, Korea University Anam Hospital, Seoul, Korea
}

Background When performing lymphovenous anastomosis, it is sometimes difficult to find venules in the proximity of an ideal lymphatic vessel that have a similar diameter to that of the lymphatic vessel. In this situation, larger venules can be used.

Methods The authors evaluated the efficacy of and patient satisfaction with lymphovenous bypass with sleeve-in anastomosis. Between January 2014 and December 2016, we performed this procedure in 18 patients (eight upper extremities and 10 lower extremities) with secondary lymphedema. Lymphovenous bypass with sleeve-in anastomosis was performed under microscopy after injecting indocyanine green dye. The circumferential diameter was measured before lymphovenous bypass and at 1, 2, and 6 months after the procedure. An outcomes survey that included patients' qualitative satisfaction with lymphovenous bypass was conducted at 6 months postoperatively.

Results Almost all patients showed quantitative improvements after surgery. The circumferential reduction rate in patients with stage II lymphedema of both the upper and lower extremities was significantly greater than in their counterparts with stage III/IV Iymphedema. The circumferential reduction rate was lower in lower-extremity patients than in upper-extremity patients.

Conclusions Lymphovenous bypass surgery with sleeve-in anastomosis in lymphedema patients is beneficial, and appears to be effective, when adequately-sized venules cannot be found in the proximity of an ideal lymphatic vessel.

Keywords Lymphedema / Microsurgery / Lower extremity / Upper extremity

Received: 3 Jul 2018 • Revised: 13 Nov $2018 \bullet$ Accepted: 14 Dec 2018

pISSN: 2234-6163 • elSSN: 2234-6171 • https://doi.org/10.5999/aps.2018.00773• Arch Plast Surg 2019;46:46-56
Correspondence: Eul-Sik Yoon Department of Plastic Surgery, Korea University Anam Hospital, 73 Inchonro, Seongbuk-gu, Seoul 02841, Korea Tel: +82-2-920-5368

Fax: +82-2-922-7437

E-mail: yesanam2@korea.ac.kr

\section{INTRODUCTION}

The term "lymphedema" refers to a specific pathological condition characterized by swelling of the soft tissue, especially the subcutaneous tissue, in the limbs due to impairment of the transportation capability of the lymphatic system or as a result of a mismatch of the lymphatic load to the transport capacity caused by obstruction or impaired development of vessels and nodes in the affected region of the body [1].

Lymphedema is a chronic and debilitating disease that causes physical and psychological morbidity in patients with the condition. Generally, the affected limb becomes swollen, heavy, fibrotic, and deformed. Patients may feel pain and become prone to infections, and typically require lifelong palliative interven- 
tions to prevent disease progression, rather than to cure the condition. Lymphedema can also place a significant financial burden on patients and society.

Lymphedema can be caused by any condition or procedure that damages the lymph nodes or lymphatic vessels. Cancer and its treatments are the most common cause of lymphedema. In breast cancer patients, $10 \%$ of patients who undergo axillary node dissection suffer from lymphedema, and the incidence is as high as $40 \%$ in patients receiving radiotherapy [2].

Many surgical approaches have been tried in an effort to treat obstructive lymphedema. In 1977, O'Brien et al. [3] introduced a microsurgical intervention (i.e., lymphaticovenous anastomosis) for the treatment of obstructive lymphedema in the extremities. The recent development of supermicrosurgery has further facilitated anastomosis of vessels of $0.3 \mathrm{~mm}$ to $0.8 \mathrm{~mm}$ in caliber [4]. Lymphovenous bypass creates a new pathway to drain excess lymphatic fluid that is trapped in lymphedematous areas into the venous circulation. It increases the transport capacity of the lymphatic fluid.

Nevertheless, it is sometimes difficult to find venules located near an ideal lymphatic vessel with a similar diameter to that of the lymphatic vessel. In this situation, large venules can be used by performing an end-to-side or sleeve-in anastomosis procedure between the larger venule and the lymphatic vessel [5]. However, the efficacy of lymphovenous bypass with the end-toside or sleeve-in technique remains uncertain.

Thus, in the present study, we sought to evaluate our experience with lymphovenous bypass employing the sleeve-in anastomosis technique in patients with extremity lymphedema secondary to cancer treatment, with a particular focus on assessing the procedure's efficacy and postoperative patient satisfaction.

\section{METHODS}

Institutional Review Board/Ethics Committee approval was obtained from the Institutional Review Board of Korea University Anam Hospital (K2018-1706-003).

A retrospective study was performed of all consecutive pa-

\section{Table 1. Clinical staging of peripheral lymphedema}

\begin{tabular}{|ll|}
\hline Stage & \multicolumn{1}{c|}{ Features } \\
\hline I I & No edema in spite of presence of lymphatic circulation anomalies \\
III & Permanent edema, spontaneously regressing with elevation \\
& Permanent edema, spontaneously not regressing with elevation, and \\
IV & ingravescent (acute erysipeloid lymphangitis) \\
V & Fibrolymphedema with "column" limb \\
\hline
\end{tabular}

tients with upper- or lower-extremity lymphedema who presented for lymphovenous bypass wherein sleeve-in anastomosis was performed between January 2014 and December 2016. Twenty-four patients with extremity lymphedema were included in the present study and underwent lymphovenous bypass at our institution. Sleeve-in anastomosis was performed in $18 \mathrm{pa}-$ tients who were not able to undergo end-to-end anastomosis because venules of a similar diameter to the identified lymphatic vessels were absent. We included all patients who underwent lymphovenous anastomosis with the sleeve-in technique due to secondary lymphedema, regardless of the primary cause.

The patients were staged according to the clinical staging system proposed by Campisi (Table 1) [1]. Patients with stages II, III, and IV lymphedema were designated as candidates for microlymphatic surgery.

Circumferential measurements of the extremities were taken at $10 \mathrm{~cm}$ above and below the lateral condyle in upper-extremity lymphedema patients, as well as at $10 \mathrm{~cm}$ above and below the patella in lower-extremity patients. Lymphedematous and unaffected limbs were assessed in all patients. The circumferential diameter was measured prior to lymphovenous bypass and at 1 , 2 , and 6 months after the procedure. The circumferential reduction rate (i.e., the rate of reduction in the circumferential diameter of the limb following the procedure) was defined as follows: (preoperative mean circumferential diameter-postoperative mean circumferential diameter)/preoperative mean circumferential diameter.

We grouped the patients according to the location of the affected limbs (upper or lower) and the severity of lymphedema (stage II or stages III/IV) and for statistical analysis.

Following patient selection, we created a novel survey that was designed to inquire regarding subjective perceptions of improvements in the affected area after the procedure and patients' overall satisfaction (Table 2). Each question was graded on a 0 to 5 scale, with higher scores indicating greater satisfaction. Questions Q1 to Q3 were related to volume, while questions Q4 to Q5 and Q6 to Q8 were related to the softness of the extremities and patients' overall satisfaction, respectively. A score
Q1. How would you rate the decrease in the size of your operated limb?

Q2. How comfortable do you feel in clothes after surgery?

Q3. Are you able to wear the clothes you like after surgery?

Q4. How would you rate the softness of your operated limb?

Q5. To what extent do you feel lighter after surgery?

Q6. How likely would you be to choose this procedure again?

Q7. What is your overall satisfaction with this procedure?

Q8. How likely are you to recommend this type of procedure? 
between 0 and 2 was regarded as indicating poor satisfaction with the procedure. Scores between 2 and 4 and between 4 and 5 were considered to indicate moderate satisfaction and high satisfaction with the procedure, respectively. The survey was administered 6 months after surgery in our outpatient clinic.

\section{Operative technique}

Prior to the procedure, lymphoscintigraphy was performed to confirm the presence of lymphatic obstruction and to evaluate the degree of the obstruction if present. Preoperative lymphoscintigraphy images were used as a guide to confirm the obstruction level and to determine the sites of incisions for lymphovenous anastomosis. If lymphatic obstruction was present below the knee or elbow level, 2- to 3-cm transverse incision lines were made at the dorsal side of the ankle or wrist area. Additional incisions were made at the pretibial area if patient lymphatic vessels were present on lymphoscintigraphy. In patients who had lymphatic vessel obstructions above the elbow or knee level, incisions were made on the flexor side of the knee or elbow area and on the dorsal side of the ankle or wrist area. The incision sites for lymphovenous bypass were marked with a pen.

General anesthesia was used in all patients but one; in this individual, local anesthesia was given instead due to a medical condition. Subsequently, the affected limbs were prepared for surgery.
Indocyanine green (ICG) dye $(0.3-0.5 \mathrm{~mL}$, indigo carmine $0.8 \%$; Korea United Pharm., Seoul, Korea) was intradermally injected into the first and second web spaces of the fingers/toes of the lymphedematous limb. An additional intradermal injection $(0.3-0.5 \mathrm{~mL})$ was performed at a location $5 \mathrm{~cm}$ to $6 \mathrm{~cm}$ distal from the possible incision sites. ICG dye assisted in the intraoperative identification of lymphatic vessels. To detect ICG fluorescence, a camera system (PENTERO 900; Carl Zeiss AG, Oberkochen, Germany) was used by the authors. A microscope was used at every step of surgery. A tourniquet was not used during the procedure.

At 15 to 20 minutes after the ICG injections, 2- to 3-cm incisions were made at the previously marked incision lines. We dissected the subdermal layer meticulously to identify the lymphatic vessels. Dissection was carefully performed for hemostasis. Healthy and intact lymphatic vessels appeared blue with ICG dye. After identifying healthy lymphatic vessels, lymphovenous anastomosis with adjacent venules was performed. Venules with larger diameters than those of the lymphatic vessels were selected to perform the bypass. The stumps of the vessels were flushed with heparin solution before anastomosis. A lymphatic vessel with surrounding adventitia was tucked into the venule by about 0.2 to $0.3 \mathrm{~mm}$ and anchored to the wall of the venule using 11-0 nylon with a 50- $\mu$ m needle (Fig. 1). Supermicrosurgical instruments were used in the anastomosis and dis-

\section{Fig. 1. Schematic image of the anastomosis technique}

A lymphatic vessel with the surrounding adventitia was tucked into the venule by about 0.2 to $0.3 \mathrm{~mm}$ and anchored to the wall of the venule using the pull-out suture technique.

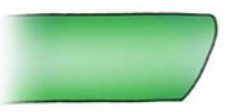

Lymphatic vessel

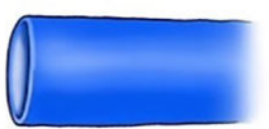

Venule
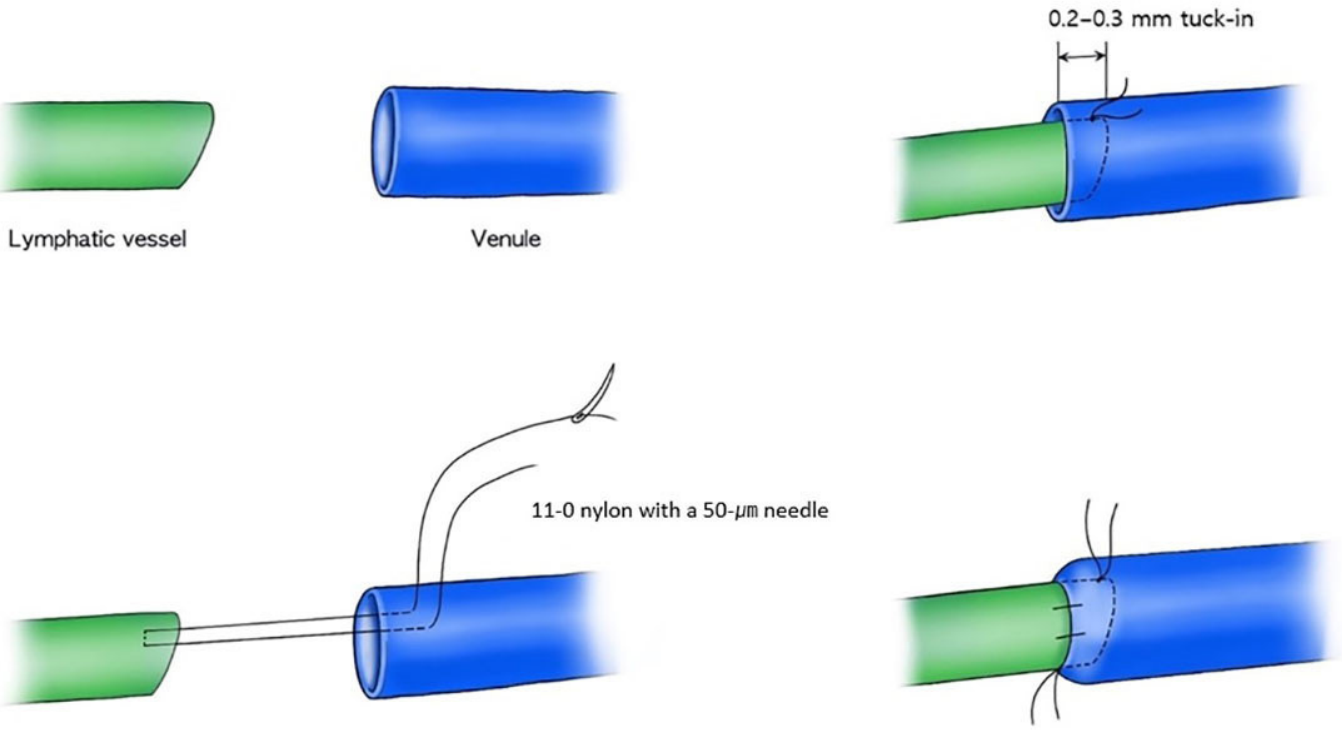

Pull-out suture for sleeve-in

Anastomosis state 
section. Three to four sutures were used for each anastomosis procedure. We confirmed patency after lymphovenous anastomosis through the microscopic observation of lymphatic fluid with ICG dye flowing through the anastomosed venule (Fig. 2).

Immediately after surgery, the operated limb was loosely compressed with an elastic bandage and elevated on a pillow. Patients were encouraged to wear compressive garments starting 1 week after surgery.

\section{Statistical analysis}

Means and standard deviations were used to summarize continuous variables. We used the independent-sample t-test to compare the percentages of quantitative improvement between different groups of patients. The analyses were performed using the SAS version 9.2 statistical software program (SAS Institute Inc., Cary, NC, USA).

\section{RESULTS}

Between January 2014 and December 2016, we performed lymphovenous bypass in 18 patients. The procedure was performed in eight upper extremities (five left and three right) and 10 lower extremities (three left and seven right). All cases of upper-extremity lymphedema were caused by breast cancer treatment. Conversely, the cases of lower-extremity lymphedema were secondary to uterine cancer $(n=3)$, cervical cancer $(n=5)$, or ovarian cancer $(n=2)$ treatment. The patients' mean age was 53 years. The mean duration of lymphedema was 4.0 years (range, 2-10 years). The patients' mean body mass index (BMI) was $23.3 \mathrm{~kg} / \mathrm{m}^{2}$ (Table 3 ).

\section{Fig. 2. Intraoperative photo of lymphovenous anastomosis}

Sleeve-in anastomosis between a lymphatic vessel and venule. The larger-caliber vessel is the venule. The black arrow indicates the anastomosis site.

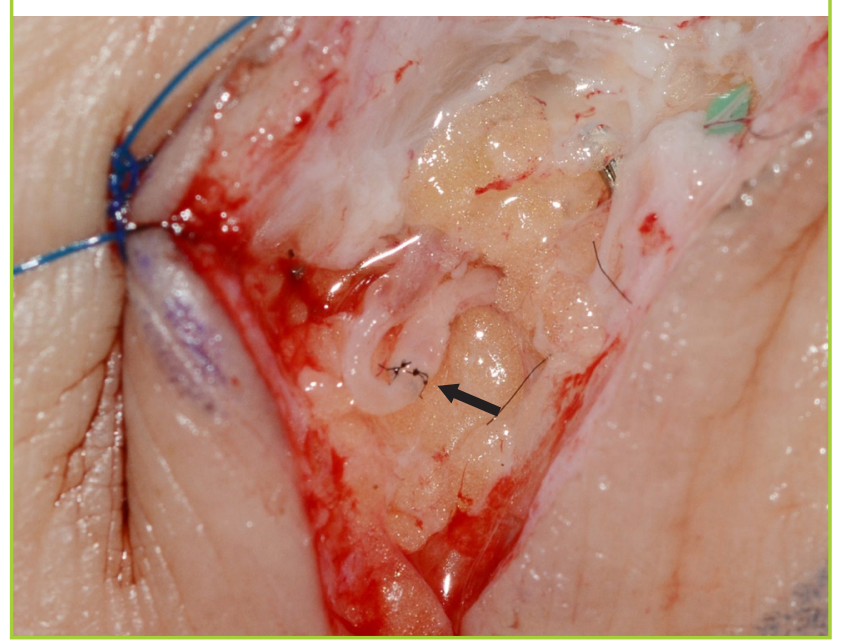

The average number of bypasses performed per patient was 2.67 (range, $2-5$ bypasses). The mean operating time was 4 hours (range, 2-5 hours). No patients experienced postoperative complications during our study.

\section{Upper extremities}

All patients showed quantitative improvements in circumferential measurements after surgery. Although the circumferential reduction rate of patients with severe (stage III/IV) (lymphede$\mathrm{ma}$ ) was not significant, these patients felt that their limbs were lighter and softer after lymphovenous anastomoses.

The duration of lymphedema was 2.7 years in the stage II group and 4.8 years in the stage III/IV group. However, the duration of disease and BMI were not significantly different between the two groups $(\mathrm{P}=0.119$ and $\mathrm{P}=0.747$, respectively) (Table 4).

The overall mean circumferential reduction rate of the upper extremities was $3.5 \%$ at 1 month, $6.4 \%$ at 2 months, and $8.3 \%$ at 6 months. Patients with stage II disease had a higher number of lymphovenous bypasses than did patients with more severe disease (i.e., stage III/IV). However, the differences were not statis-

\section{Table 3. Summary of demographics}

\begin{tabular}{|c|c|c|}
\hline Variable & Upper extremity & Lower extremity \\
\hline No. of patients & 8 & 10 \\
\hline Age $(y r)^{a)}$ & 51 & 55 \\
\hline Sex (male:female) & $0: 8$ & $0: 10$ \\
\hline BMI $\left(\mathrm{kg} / \mathrm{m}^{2}\right)^{\mathrm{a})}$ & 23.0 & 23.6 \\
\hline \multicolumn{3}{|c|}{ Campisi clinical stage } \\
\hline Stage II & 3 & 4 \\
\hline Stage III or IV & 5 & 6 \\
\hline Duration (yr) ${ }^{\mathrm{a})}$ & 4.0 & 3.9 \\
\hline Cause & Breast cancer $(n=8)$ & $\begin{array}{l}\text { Cervical cancer }(n=5) \text {, } \\
\text { uterine cancer }(n=3) \\
\text { ovarian cancer }(n=2)\end{array}$ \\
\hline
\end{tabular}

Table 4. Results of upper-extremity lymphedema patients according to disease severity

\begin{tabular}{|c|c|c|c|}
\hline Characteristic & $\begin{array}{c}\text { Stage II } \\
(n=3)\end{array}$ & $\begin{array}{l}\text { Stage III or IV } \\
(n=5)\end{array}$ & P-value \\
\hline Duration of lymphedema $(\mathrm{yr})^{\mathrm{a})}$ & 2.7 & 4.8 & 0.119 \\
\hline BMI $\left(\mathrm{kg} / \mathrm{m}^{2}\right)^{\mathrm{a})}$ & 23.3 & 22.9 & 0.747 \\
\hline No. of bypasses ${ }^{\text {b) }}$ & 3.3 & 2.4 & 0.747 \\
\hline \multicolumn{4}{|l|}{ Reduction rate (\%) } \\
\hline 1 Month & 4.2 & 3.1 & 0.206 \\
\hline 2 Months & 8.3 & 5.3 & 0.004 \\
\hline 6 Months & 10.5 & 6.9 & 0.016 \\
\hline
\end{tabular}


tically significant between the two groups. Upper-extremity stage II lymphedema patients showed a higher circumferential reduction rate than patients with severe lymphedema. This difference was statistically significant at 2 months and 6 months, but not at 1 month (Tables 4 and 5).

The mean circumferential reduction rate in patients with stage II disease was $4.2 \%, 8.3 \%$, and $10.5 \%$ at 1,2 , and 6 months, respectively. The mean circumferential reduction rate in patients with stage III/IV disease was $3.1 \%$ at 1 month, $5.3 \%$ at 2 months, and $6.9 \%$ at 6 months (Table 5).

\section{Lower extremities}

Each of the 10 patients who underwent lower-extremity lymphovenous bypass noted a slight volume reduction after the procedure. The overall circumferential reduction rate of the lower extremities was $3.7 \%$ at 1 month, $4.6 \%$ at 2 months, and $4.9 \%$ at 6 months. The overall circumferential reduction rate was lower in lower-extremity patients than in upper-extremity patients (Table 5).

The mean lymphedema duration was 3.5 years in stage II patients and 4.2 years in stage III/IV patients $(\mathrm{P}=0.684)$. The du-

\begin{tabular}{|c|c|c|c|c|}
\hline Stage & Months & $\begin{array}{c}\text { Upper } \\
\text { extremities } \\
(\%)\end{array}$ & $\begin{array}{l}\text { Lower } \\
\text { extremities } \\
(\%)\end{array}$ & P-value \\
\hline \multirow[t]{3}{*}{ Stage II } & 1 & 4.2 & 4.2 & 0.979 \\
\hline & 2 & 8.3 & 5.0 & 0.004 \\
\hline & 6 & 10.5 & 5.4 & 0.034 \\
\hline \multirow[t]{3}{*}{ Stage III or IV } & 1 & 3.1 & 3.3 & 0.691 \\
\hline & 2 & 5.3 & 4.3 & 0.043 \\
\hline & 6 & 6.9 & 4.5 & 0.002 \\
\hline \multirow[t]{3}{*}{ Overall } & 1 & 3.5 & 3.7 & 0.722 \\
\hline & 2 & 6.4 & 4.6 & 0.025 \\
\hline & 6 & 8.3 & 4.9 & 0.003 \\
\hline
\end{tabular}

Table 6. Results of lower-extremity lymphedema patients according to disease severity

\begin{tabular}{|lccc|}
\hline Characteristic & $\begin{array}{c}\text { Stage II } \\
(\mathbf{n}=\mathbf{4})\end{array}$ & $\begin{array}{c}\text { Stage III or IV } \\
(\mathbf{n}=\mathbf{6})\end{array}$ & P-value \\
\hline Duration of lymphedema (yr) $)^{\text {a) }}$ & 3.5 & 4.2 & 0.684 \\
BMI (kg/m2) & 22.3 & 24.5 & 0.137 \\
No. of bypasses $)^{\text {b) }}$ & 3.0 & 2.3 & 0.312 \\
Reduction rate (\%) & & & \\
1 Month & 4.2 & 3.3 & 0.165 \\
2 Months & 5.0 & 4.3 & 0.065 \\
6 Months & 5.4 & 4.5 & 0.037 \\
\hline BMl, body mass index. & & & \\
a'Mean; ${ }^{\text {b)Average. }}$ & & & \\
\hline
\end{tabular}

ration of disease and $\mathrm{BMI}$ were not significantly different between the two groups $(\mathrm{P}=0.684$ and $\mathrm{P}=0.137$, respectively). Stage II patients underwent a higher number of lymphovenous bypass procedures than patients with more severe disease, but the difference was not statistically significant $(\mathrm{P}=0.312)$.

In patients with stage II lower-extremity lymphedema, the circumferential reduction rate was $4.2 \%$ at 1 month, $5.0 \%$ at 2 months, and 5.4\% at 6 months. Patients with stage III/IV lymphedema showed a circumferential reduction rate of $3.3 \%$ at 1 month, $4.3 \%$ at 2 months, and $4.5 \%$ at 6 months (Table 5 ). As in upper-extremity lymphedema patients, patients with stage II lower-extremity lymphedema showed a greater circumferential reduction rate than did patients with stage III/IV lower-extremity lymphedema. This difference was only statistically significant at 6 months after surgery (Table 6).

In our study, a greater decrease in excess volume was observed in the upper extremities than in the lower extremities. This difference was statistically significant at 2 months and 6 months after surgery in stage II patients, stage III/IV patients, and the overall patient cohort. Upper-extremity stage II patients showed the greatest circumferential reduction rate among the four groups, followed by upper-extremity stage III/IV, lower-extremity stage II, and lower-extremity stage III/IV patients (Fig. 3).

\section{Patient satisfaction}

The response rate of the satisfaction questionnaire was $94.4 \%$ $(17 / 18)$. For the volume-related questions (Q1-Q3), the upper-extremity stage II lymphedema group had higher scores than the other groups for the decrease in size of the limb that

\section{Fig. 3. Circumferential reduction rates}

Circumferential reduction rates of upper- and lower-extremity lymphedema patients at 1, 2, and 6 months after lymphovenous bypass.

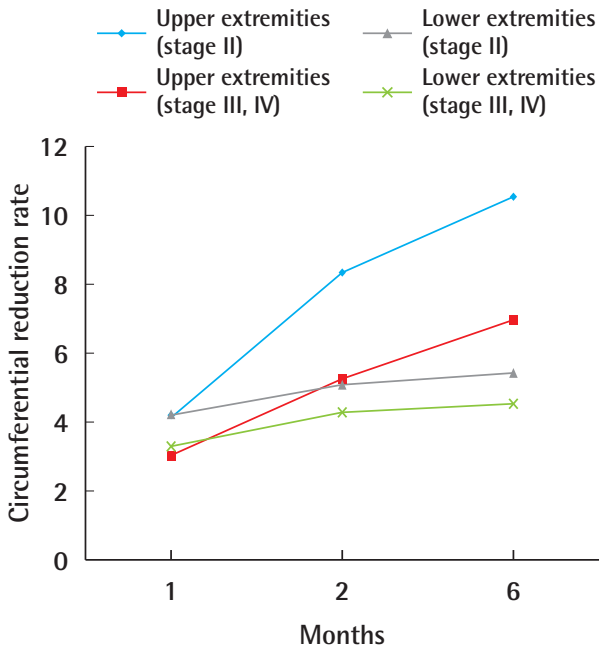


was operated upon, as well as comfort in clothes and the ability to wear the clothes that patients desired. Stage II lymphedema patients had higher scores than stage III/IV lymphedema patients, and this was the case for both the upper-extremity and lower-extremity patients. No significant differences in comfort in clothes and the ability to wear the clothes that patients desired were detected between the upper-extremity lymphedema (both stages II and III/IV) and the lower-extremity lymphedema patients (both stages II and III/IV). However, the upper-extremity lymphedema patients had a higher level of satisfaction with the decrease in size of the operated-upon limb than the lower-extremity lymphedema patients.

The limb softness-related questions (Q4 and Q5) showed that the lower-extremity stage III/IV lymphedema patients had the lowest scores for softness and lightness of the operated-upon limb. There were no significant differences among the upperextremity stage II, upper-extremity stage III/IV, and lower-extremity stage II groups, with all having scores $>3.0$ (Fig. 4).

For the overall satisfaction questions (Q6-Q8), upper-extremity lymphedema patients (stages II and III/IV) showed higher scores than lower-extremity lymphedema patients (stages II and III/IV) in terms of the likelihood that they would choose to undergo the procedure again, overall satisfaction with lymphovenous bypass, and the likelihood that they would recommend this procedure to others. Stage II lymphedema patients showed higher satisfaction than stage III/IV lymphedema patients, and this was the case for both the upper- and lower-extremity patients. Upper-extremity stage II patients had the highest scores for the three satisfaction-related questions, and lowerextremity stage III/IV patients had the lowest scores.

The upper-extremity stage II lymphedema group had the highest scores for almost all questions, except softness of the op- erated-upon limb. The softness of the operated-upon limb in the upper-extremity stage II group was rated as 4.0, indicating a high level of satisfaction. In the upper-extremity stage III/IV lymphedema group, the scores for softness, lightness, and overall satisfaction indicated moderate satisfaction $(<3.0)$. In addition, these patients reported moderate satisfaction on the volume-related questions, with scores between 2.0 and 3.0.

Lower-extremity stage II lymphedema patients showed moderate overall satisfaction and moderate scores for the softnessrelated questions. However, for the volume-related questions, although the clothing-related scores were moderately satisfactory, the decrease in size received a score indicating poor satisfaction. The lower-extremity stage III/IV group showed the lowest scores for all questions. Patients with lower-extremity stage III/ IV lymphedema reported moderate overall satisfaction and moderate scores for the softness-related questions but indicated poor satisfaction on the volume-related questions.

\section{Case reports}

Case 1 (patient 5)

A 55-year-old woman with secondary lymphedema of the left leg of 3 years' duration had experienced ineffective conservative treatment. She had undergone pelvic lymph node dissection due to cervical cancer. The mild lymphedema of the left leg was classified as stage II. Four lymphovenous anastomoses were made with two small skin incisions on the anterior aspect of the ankle and popliteal area. Constant compressive therapy for 6 months after the operation gradually reduced the circumferential diameter. The preoperative circumferential diameters were $45 \mathrm{~cm}$ above the knee level and $36.5 \mathrm{~cm}$ below the knee level. The postoperative circumferential diameter above the knee level was $44 \mathrm{~cm}$ at 1 month, $43.5 \mathrm{~cm}$ at 2 months, and $43 \mathrm{~cm}$ at 6

\section{Fig. 4. Patient survey responses}

Note that the question labels (01-08) correlate with the questions in Table 2.

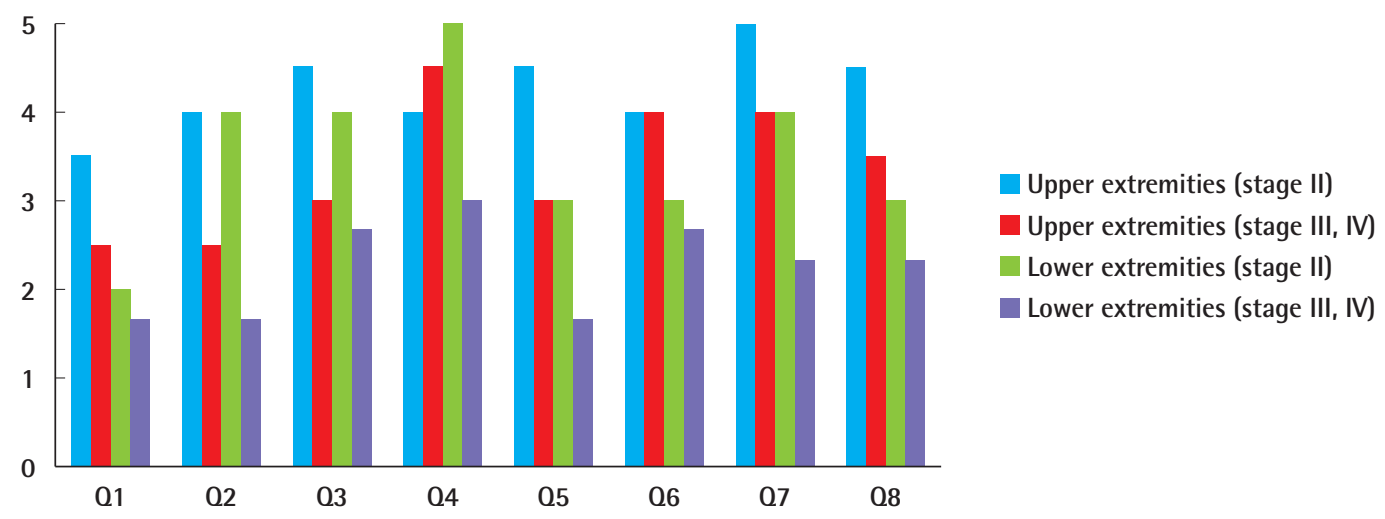




\section{Fig. 5. Case 1}

A 55-year-old woman with stage II lymphedema of the left leg for 3 years. The patient underwent lymphovenous bypass on the anterior aspect of the ankle and popliteal area. (A) Preoperative photography. (B) Six months after surgery. A postoperative decrease in circumference was noted in the left leg.
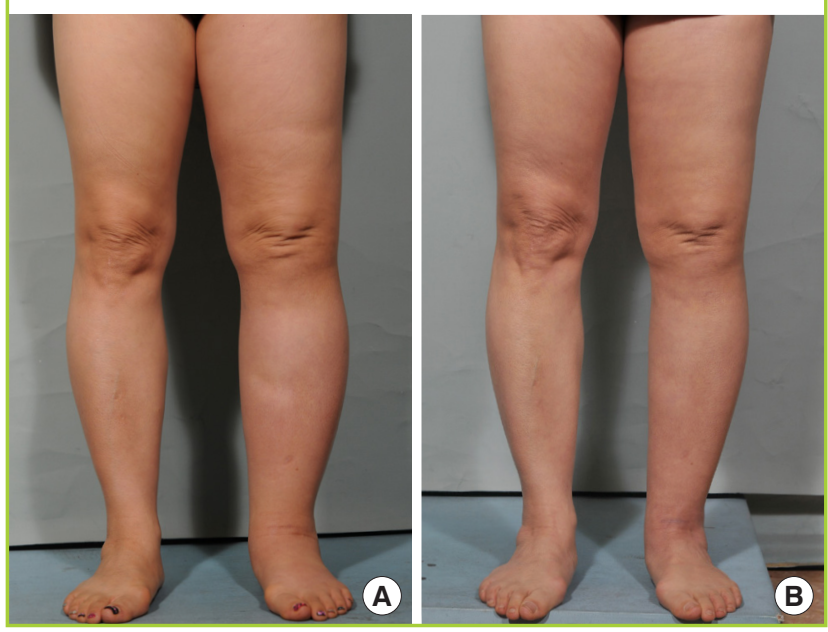

months. The postoperative circumferential diameter below the knee level was $36 \mathrm{~cm}$ at 1 month, $36 \mathrm{~cm}$ at 2 months, and 35.5 $\mathrm{cm}$ at 6 months. The circumferential reduction rate was $3.8 \%$ at 1 month, $4.4 \%$ at 2 months, and $5 \%$ at 6 months (Fig. 5).

\section{Case 2 (patient 6)}

A 61-year-old woman had stage III lymphedema of the left leg. The edema had continued for 1.5 years after an endometrial cancer operation, during which time she continuously wore elastic stockings. Three lymphovenous anastomoses were performed through three incisions. The incisions were made at the medial thigh area, below the knee, and at the anterior aspect of the ankle, respectively. She used compressive stockings postoperatively. The preoperative circumferential diameters were 38 $\mathrm{cm}$ above the knee level and $34 \mathrm{~cm}$ below the knee level. The postoperative circumferential diameter above the knee level was $33 \mathrm{~cm}$ at 1 month, $32 \mathrm{~cm}$ at 2 months, and 32 at 6 months. The postoperative circumferential diameter below the knee level was $31.5 \mathrm{~cm}$ at 1 month, $29 \mathrm{~cm}$ at 2 months, and $28 \mathrm{~cm}$ at 6 months. The final circumferential reduction rate at 6 months was $17.6 \%$. She felt lighter and softer after lymphovenous bypass (Fig. 6).

\section{Case 3 (patient 1)}

A 59-year-old woman had stage III lymphedema of the left arm for a duration of 7 years. She rarely used elastic stockings during this period. She had undergone axillary lymph node dissection 9 years previously because of left breast cancer. Two lymphovenous anastomoses were performed at the anterior aspect of the elbow. The preoperative circumferential diameters were 40

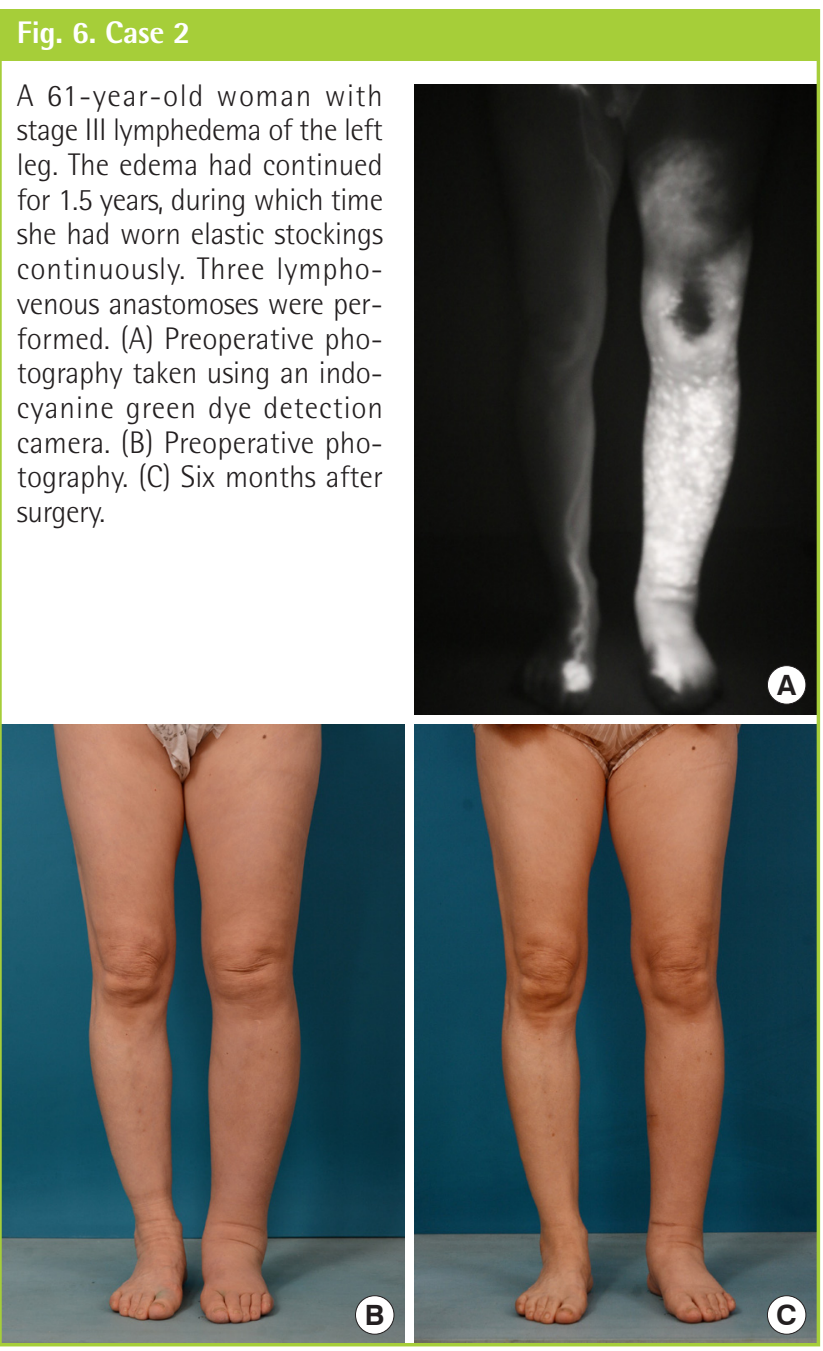

$\mathrm{cm}$ above the elbow level and $30 \mathrm{~cm}$ below the elbow level. The postoperative circumferential diameter above the elbow level was $38 \mathrm{~cm}$ at 1 month, $37 \mathrm{~cm}$ at 2 months, and $37 \mathrm{~cm}$ at 6 months. The postoperative circumferential diameter below the elbow level was $29 \mathrm{~cm}$ at 1 month, $28 \mathrm{~cm}$ at 2 months, and 27.5 $\mathrm{cm}$ at 6 months. The circumferential reduction rate was $3.8 \%$ at 1 month, $6.3 \%$ at 2 months, and $6.9 \%$ at 6 months (Fig. 7 ).

\section{DISCUSSION}

The presentation and progress of lymphedema depends on many variables, including the etiology, stage, and extent of edema, as well as the affected anatomic region. The treatment of lymphedema varies depending on its symptoms and severity. When minor pain and tissue swelling occur, palliative compression methods such as compression lymphatic massage or compression garments can be used. Furthermore, surgical approaches such as lymph node transfer and lymphovenous bypass can be applied in patients who are resistant to nonoperative treat- 


\section{Fig. 7. Case 3}

A 59-year-old woman with stage III left arm lymphedema for a duration of 7 years following left mastectomy and lymph node dissection. She rarely used elastic stockings during this period. Two lymphovenous anastomoses were performed at the anterior aspect of the elbow. (A) Preoperative photography. (B) Two months after surgery. (C) Six months after surgery.
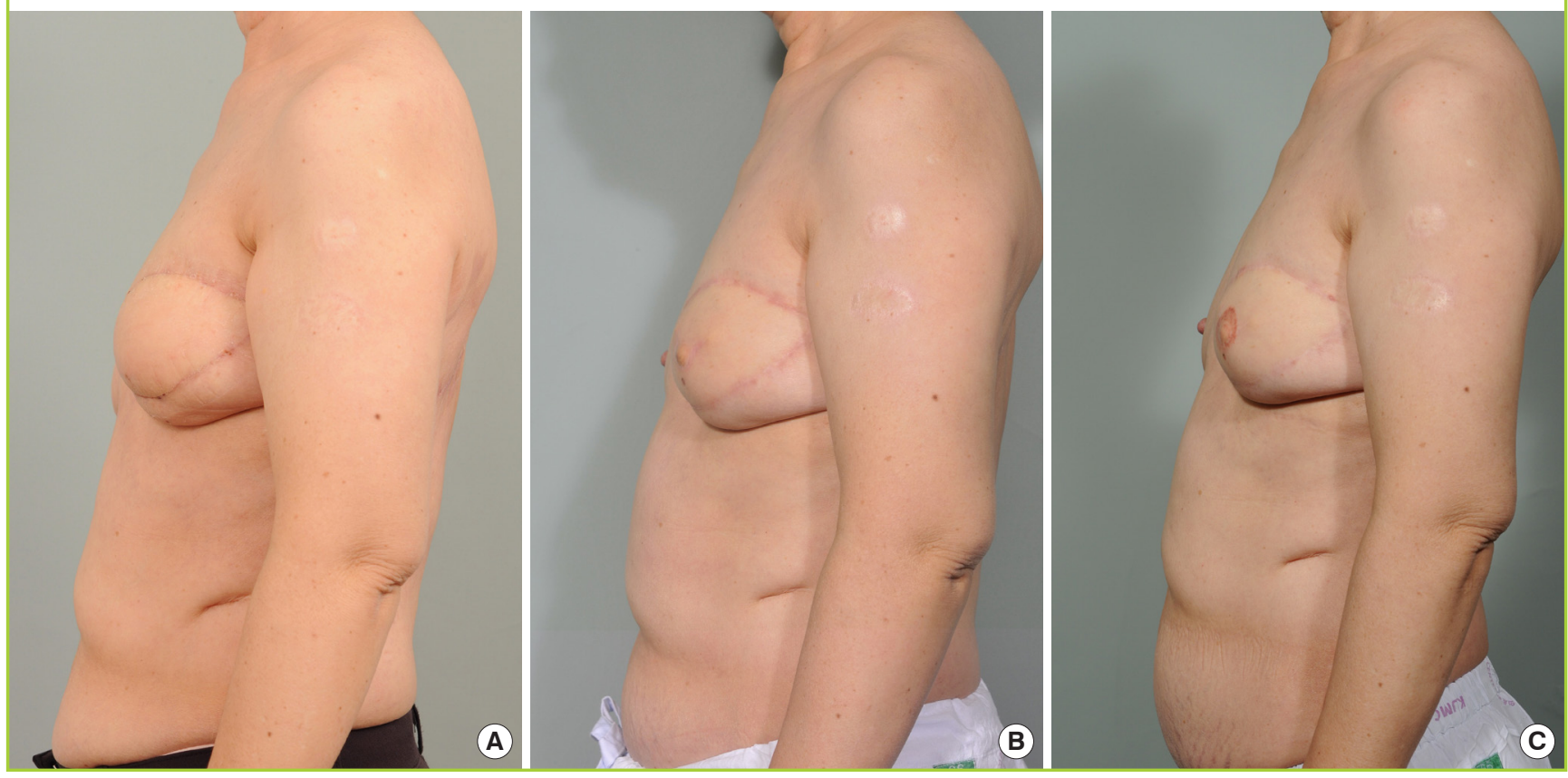

ments.

Both nonoperative and surgical treatments of lymphedema aim to decrease the size of the edematous area, improve skin texture, and reduce episodes of infection [6]. An elastic stocking or bandage, which is commonly accepted as the mainstay of lymphedema treatment, is used to prevent the exacerbation of edema by applying external force and increasing tissue pressure. The elastic bandage must always be worn and the edema undergoes very slow reduction during compression therapy. These limitations of compression therapy lead to poor patient tolerance of the treatment. However, even patients who undergo surgical treatments, such as lymphovenous bypass or free vascularized lymph node transfer, are required to wear compressive bandages after surgery to prevent the recurrence of edema. In our study, compression therapy continued for 1 week after surgery and all patients were encouraged to wear compressive stockings.

A clinical situation that is sometimes observed in the operating room is that appropriate lymphatic vessels are present in the surgical bed, but recipient venules are absent. In this case, it is possible to choose larger venules or veins situated in close proximity. Such vessels are usually located deeper in the subdermal layer or subcutaneous layer. Anastomosis is then performed via a sleeve-in or end-to-side method, since performing end-to-end anastomosis is not possible. However, the efficacy of lymphovenous bypass with the sleeve-in technique is still uncertain ac- cording to the literature.

Regarding the postoperative decrease in circumference, upperextremity stages II and III/IV lymphedema patients showed mean circumferential reduction rates of $10.5 \%$ and $6.9 \%$, respectively, which corresponded to reductions of $4.5 \mathrm{~cm}$ and 2.5 $\mathrm{cm}$ in circumferential diameter at 6 months after surgery. In lower-extremity lymphedema patients, the mean reduction rates of those with stages II and III/IV disease were 5.4\% and 4.5\%, respectively, corresponding to reductions of $2 \mathrm{~cm}$ and $1.7 \mathrm{~cm}$, respectively. In 2003, Koshima et al. [7] analyzed lymphedema of the lower extremity in 13 patients who underwent end-toend lymphovenous anastomosis. The authors reported that the average decrease in circumference was $4.7 \mathrm{~cm}$. In addition, Auba et al. [8] evaluated 12 patients, five with lower-limb and seven with upper-limb lymphedema, who underwent end-to-end lymphovenous anastomosis. They reported a $0.45 \mathrm{~cm}$ circumferential reduction in the lower extremities and a $0.85 \mathrm{~cm}$ circumferential reduction in the upper extremities. The amount of reduction in the lymphedematous limb after lymphovenous bypass varies among studies because of differences in the severity of patients and the measurement methods used. It is difficult to draw definitive conclusions about whether the end-to-end or sleeve-in anastomosis technique is superior in terms of efficacy, although it appears that sleeve-in anastomosis has similar efficacy to that of end-to-end anastomosis and can be effectively used 
in patients lacking appropriately sized venules.

In 2003, Yamamoto et al. [9] evaluated the efficacy of microsurgical lymphaticovenous implantation in 18 patients with upper-limb lymphedema. Microsurgical lymphaticovenous implantation is a similar technique to sleeve-in anastomosis, which was used in our study. They reported that the average decreases in circumference were $3.7 \mathrm{~cm}$ at the distal site and $3.6 \mathrm{~cm}$ at the proximal site. However, they did not report efficacy according to the severity of lymphedema; rather, they simply analyzed the relationship between the preoperative diameter discrepancy and postoperative improvement. In our study, the average decreases in circumference were $4.5 \mathrm{~cm}$ in stage II upper-limb patients and $2.5 \mathrm{~cm}$ in stage III/IV upper-limb patients. The average reduction rate of stage II patients in our study was higher than in Yamamoto's study, while the reduction rate of stage III/ IV patients was lower.

Lymphovenous bypass was less effective for lower-extremity lymphedema than for upper-extremity lymphedema. In 1990, O'Brien et al. [6] presented long-term results of lymphovenous anastomosis, reporting that excess volume decreased more frequently in the upper extremities than in the lower extremities. In 2013, Chang et al. [10] described 100 cases of lymphovenous bypass in the extremities and reported that only four of seven patients experienced symptom improvement and that volume reduction occurred in only two patients. Similar results were obtained in our study. The circumferential reduction rate of the lower extremities in our study at 6 months was $5.4 \%$ in stage II patients and $4.5 \%$ in stage III/IV patients. The circumferential reduction rate of lower-extremity patients was lower than that of upper-extremity patients. Gravity is thought to play an important role in lymphedema by causing difficulty in returning lower-extremity lymphatic fluid to the heart, which exacerbates lower-extremity lymphedema and impairs the function of lymphovenous bypass.

The circumferential reduction rates in the lymphedematous limbs of patients with stage II lymphedema of both the upper and lower extremities were significantly greater than those their counterparts with stage III/IV lymphedema. These findings indicate that lymphovenous bypass is more effective for decreasing volume in cases of early-stage lymphedema than in cases of late-stage lymphedema. Many surgeons believe that microlymphatic surgery should be performed at an early stage of lymphedema, before the superficial lymphatics are extensively damaged by increased pressure and while the distal lymphatics are still patent and functioning due to preserved smooth muscle function $[11,12]$. However, it is difficult to estimate the condition and residual function of the lymphatic vessels preoperatively. Physical examination and lymphoscintigraphic findings do not correlate well with the condition of the residual lymphatic vessels. Moreover, the duration of lymphedema does not necessarily correlate with its severity [13]. In our experience, the staging system proposed by Campisi [1] seems to appropriately reflect the residual function of lymphatic vessels, because lowerstage patients had better results after surgery (Table 1).

Nagase et al. [14] reported that lymphovenous anastomosis can still be applied in patients with late-stage lymphedema, even if the smooth muscle is damaged. However, patients need compression therapy to discharge the lymphatic fluid into the venules. Therefore, microlymphatic surgery is not limited to the early stage, and it may be combined with nonoperative treatment in select cases of later stages of lymphedema. However, in patients with stage III/IV lymphedema, few lymphatic vessels are available for anastomosis, and patients with severe disease typically already have tissue fibrosis [10]. Thus, if available lymphatic vessels are present in the operating field, lymphovenous anastomosis is a viable treatment choice, whereas if there are no available lymphatic vessels, then vascularized lymph node transfer should be considered instead.

Although all patients with stage III/IV lymphedema in our study had available lymphatic vessels for lymphovenous anastomosis, the number of available lymphatic vessels was lower in more severe cases of lymphedema. After lymphovenous bypass, patients with later-stage lymphedema were encouraged to wear compressive stockings and showed a slight volume reduction after surgery.

ICG lymphangiography is a useful method to evaluate the severity of the patient's condition in preparation for lymphovenous anastomosis surgery [15]. Lymphatic vessels can be imaged by an infrared camera system after injecting ICG dye intradermally into the web spaces of the hand or foot. This system is helpful for mapping lymphatic vessels intraoperatively and for accurately identifying functioning lymphatic vessels for bypass. However, since an ideal infrared camera system was not available at our institution, we additionally injected ICG dye at a site located $5 \mathrm{~cm}$ to $6 \mathrm{~cm}$ distally from the possible incision line. Functioning lymphatic vessels were stained with ICG dye after this additional injection. This method is more beneficial than a random exploration of lymphatic vessels without an additional injection.

The authors acknowledge a number of limitations of the current study. In this series, the follow-up period after surgery was short. Almost all study patients experienced volume reduction at 6 months after surgery. However, whether the volume will decrease more as time goes on or whether lymphedema will relapse is uncertain. Lee et al. [16] reported two cases of lymphedema with no significant reduction in limb circumference over 
a 1-year follow-up period because lymphovenous anastomosis site patency did not persist long enough as a result of scarring at the anastomosis site following reduction of tissue pressure. Puckett et al. [17] stated that the most likely reason for occlusion of the anastomosis was the regurgitation of venous blood through the anastomosis into the lymphatic vessel, because the vein used for anastomosis can lack valves. However, Chang et al. [10] reported a $35 \%$ volume reduction at 2 years and a $38 \%$ reduction at 3 years in 10 patients with upper-extremity lymphedema. The long-term effects of lymphovenous anastomosis are still controversial and future studies are needed. Finally, our sample size was too small to show statistically significant differences between groups, and therefore may not have adequately represented the efficacy of lymphovenous bypass with the sleeve-in method in this broadening population of patients. However, we feel that reporting the circumferential reduction rate of the sleeve-in anastomosis technique according to the stage of lymphedema is of paramount importance.

To our knowledge, the present study is the first report containing an evaluation of patients' satisfaction following lymphovenous bypass with the sleeve-in method using a survey. Satisfaction was higher in patients with lower-stage disease than in those with higher-stage disease, and in those with upper-extremity lymphedema than in those with lower-extremity lymphedema. Although satisfaction with the decrease in size after surgery was low in patients with lower-extremity lymphedema, they still reported feeling lighter and softer after surgery, according to our survey.

In conclusion, lymphovenous bypass surgery with the sleevein method in lymphedema patients is beneficial when adequately sized venules are absent in the proximity of an ideal lymphatic vessel. Moreover, lymphovenous bypass with sleeve-in anastomosis is effective, especially in the early stage of upper-extremity lymphedema. Although lymphovenous bypass in patients with severe lower-extremity lymphedema does not effectively lead to a decrease in the size of the affected limb, these patients still expressed satisfaction with the lightness and softness of the lymphedematous limb after lymphovenous anastomosis. The current results are preliminary, and longer-term results and verification of our findings by other studies are needed to further popularize this treatment for lymphedema.

\section{NOTES}

\section{Conflict of interest}

No potential conflict of interest relevant to this article was reported.

\section{Ethical approval}

The study was approved by the Institutional Review Board of Korea University Anam Hospital (IRB No. K2018-1706-003) and performed in accordance with the principles of the Declaration of Helsinki. Written informed consents were obtained.

\section{Patient consent}

The patients provided written informed consent for the publication and the use of their images.

\section{ORCID}

Jae-Ho Chung https://orcid.org/0000-0002-8351-2444

Si-Ook Baek https://orcid.org/0000-0003-3896-5572

Ho-Jin Park https://orcid.org/0000-0001-9809-0558

Byung-Il Lee https://orcid.org/0000-0001-8183-0388

Seung-Ha Park https://orcid.org/0000-0002-2667-2791

Eul-Sik Yoon https://orcid.org/0000-0001-5734-6625

\section{REFERENCES}

1. Campisi C. Lymphoedema: modern diagnostic and therapeutic aspects. Int Angiol 1999;18:14-24.

2. Sakorafas GH, Peros G, Cataliotti L, et al. Lymphedema following axillary lymph node dissection for breast cancer. Surg Oncol 2006;15:153-65.

3. O’Brien BM, Chait LA, Hurwitz PJ. Microlymphatic surgery. Orthop Clin North Am 1977;8:405-24.

4. Koshima I, Inagawa K, Urushibara K, et al. Paraumbilical perforator flap without deep inferior epigastric vessels. Plast Reconstr Surg 1998;102:1052-7.

5. Lasso JM, Perez Cano R. Practical solutions for lymphaticovenous anastomosis. J Reconstr Microsurg 2013;29:1-4.

6. O’Brien BM, Mellow CG, Khazanchi RK, et al. Long-term results after microlymphaticovenous anastomoses for the treatment of obstructive lymphedema. Plast Reconstr Surg 1990;85:562-72.

7. Koshima I, Nanba Y, Tsutsui T, et al. Long-term follow-up after lymphaticovenular anastomosis for lymphedema in the leg.J Reconstr Microsurg 2003;19:209-15.

8. Auba C, Marre D, Rodriguez-Losada G, et al. Lymphaticovenular anastomoses for lymphedema treatment: 18 months postoperative outcomes. Microsurgery 2012;32:261-8.

9. Yamamoto Y, Horiuchi K, Sasaki S, et al. Follow-up study of upper limb lymphedema patients treated by microsurgical lymphaticovenous implantation (MLVI) combined with compression therapy. Microsurgery 2003;23:21-6.

10. Chang DW, Suami H, Skoracki R. A prospective analysis of 100 consecutive lymphovenous bypass cases for treatment 
of extremity lymphedema. Plast Reconstr Surg 2013;132: 1305-14.

11. Koshima I, Inagawa K, Urushibara K, et al. Supermicrosurgical lymphaticovenular anastomosis for the treatment of lymphedema in the upper extremities. J Reconstr Microsurg 2000;16:437-42.

12. Huang GK, Hu RQ, Liu ZZ, et al. Microlymphaticovenous anastomosis in the treatment of lower limb obstructive lymphedema: analysis of 91 cases. Plast Reconstr Surg 1985; 76:671-85.

13. Franks PJ, Moffatt CJ, Doherty DC, et al. Assessment of health-related quality of life in patients with lymphedema of the lower limb. Wound Repair Regen 2006;14:110-8.

14. Nagase $T$, Gonda $K$, Inoue $K$, et al. Treatment of lymphede- ma with lymphaticovenular anastomoses. Int J Clin Oncol 2005;10:304-10.

15. Yamamoto T, Narushima M, Doi K, et al. Characteristic indocyanine green lymphography findings in lower extremity lymphedema: the generation of a novel lymphedema severity staging system using dermal backflow patterns. Plast Reconstr Surg 2011;127:1979-86.

16. Lee YJ, Park JI, Han HS, et al. Treatment of the lymphedema using microlymphaticovenous anastomoses: two cases. J Korean Soc Plast Reconstr Surg 1992;19:1041-8.

17. Puckett CL, Jacobs GR, Hurvitz JS, et al. Evaluation of lymphovenous anastomoses in obstructive lymphedema. Plast Reconstr Surg 1980;66:116-20. 